VOL. I (1969), 425-430.

\title{
On the relationship between graphs and partially balanced incomplete block designs
}

\author{
W. D. Wallis
}

Certain theorems which are already known show that if a partially balanced incomplete block design with suitable parameters exists then there is a $(V, K, \Lambda)$-graph. We prove that the existence of such a graph is in fact equivalent to the existence of a certain partially balanced design. The known necessary conditions for $(V, K, \Lambda$-graphs then follow from well-known necessary conditions for designs.

We also establish the existence of a new class of these graphs.

1. Partially balanced designs and graphs

Suppose $G$ is a graph on $V$ points. Write $S_{x}$ for the set of all points joined to the point $P_{x}$ by arcs of $G$. If $\left|S_{x}\right|=K$ and $\left|S_{x} \cap S_{y}\right|=\Lambda$ for any $x$ and $y$, then $G$ is a $(V, K, \Lambda)$-graph (see [1] and [10]). The points $P_{x}$ and sets $S_{x}$ form a $(V, K, \Lambda)$-configuration if we interpret the points as treatments and sets as blocks.

A partially balanced incomplete block design with two classes of associativity, or $P B I B D(2)$, is a scheme for arranging $v$ objects (treatments) into $b$ sets (blocks) of size $k$, with each treatment a member of $r$ blocks. Any two treatments are called either first or second associates; the number $n_{i}$ of treatments $P$ such that $P_{x}$ and $P$ are $i$-th associates must be independent of $x$; given a pair $\left\{P_{x}, P_{y}\right\}$ of $i$-th associates, there must be $\lambda_{i}$ blocks which contain both $P_{x}$ and $P_{y}$,

Received 9 September 1969. 
and $p_{j k}^{i}$ treatments $Q$ such that $\left\{P_{x}, Q\right\}$ and $\left\{P_{y}, Q\right\}$ are $j-$ th and $k$-th associates respectively.

We have defined sixteen constants related to a $P B I B D$ (2); there are various relations between these parameters [3, p. 213]:

$$
\left\{\begin{array}{l}
p_{11}^{1}+p_{12}^{1}+1=p_{11}^{2}+p_{12}^{2}=n_{1} \\
p_{21}^{2}+p_{22}^{2}+1=p_{21}^{1}+p_{22}^{1}=n_{2} \\
p_{12}^{1}=p_{21}^{1}, p_{12}^{2}=p_{21}^{2}, \\
n_{1} p_{12}^{1}=n_{2} p_{11}^{2}, n_{1} p_{22}^{1}=n_{2} p_{12}^{2}
\end{array}\right.
$$

enable us to define all the $p_{j k}^{i}$ in terms of $p_{11}^{1}$, while the other parameters must satisfy

$$
\left\{\begin{aligned}
v r & =b k, \\
n_{2} & =v-n_{1}-1, \\
\lambda_{1} n_{1}+\lambda_{2} n_{2} & =r(k-1) .
\end{aligned}\right.
$$

We will indicate the parameter scheme of a $P B I B D(2)$ by the 8-tuple

$$
\left(v, r, k ; \lambda_{1}, \lambda_{2} ; n_{1} ; p_{11}^{1}, p_{11}^{2}\right),
$$

the inclusion of $p_{11}^{2}$ and $\lambda_{2}$ being redundant but convenient.

THEOREM 1. If there is a PBIBD (2) which satisfies $p_{11}^{1}=p_{11}^{2}$, then there is a $(V, K, \Lambda)$-graph with parameters

$$
\begin{aligned}
& V=v \\
& K=n_{1} \\
& \Lambda=p_{11}^{1}=p_{11}^{2} .
\end{aligned}
$$

Proof. Take the treatments in the design as points of a graph; two points are joined if and only if they are first associates. This graph satisfies the conditions for a $(V, K, \Lambda)$-graph with the given parameters.

THEOREM 2. If there is a $P B I B D(2)$ which satisfies $p_{22}^{1}=p_{22}^{2}$, then there is a $(V, K, \Lambda)$-graph with parometers

$$
\begin{aligned}
& V=v \\
& K=n_{2} \\
& \Lambda=p_{22}^{1}=p_{22}^{2} .
\end{aligned}
$$


The proof is as for Theorem 1, except that second associates are joined.

Bose [2] has shown that a graph is obtained on joining the first associates of a $P B I B D(2)$; theorems essentially the same as our Theorems 1 and 2 are used in [9] to construct $(V, K, \Lambda)$-configurations. However, the two ideas have not been put together before.

THEOREM 3. There is a $(V, K, \Lambda)$-graph if and only if there is a $P B I B D(2)$ with parameters

$$
(V, K, 2 ; 1,0 ; K ; \Lambda, \Lambda) \text {. }
$$

Proof. The "if" part follows from Theorem 1 . Now suppose there is a $(V, K, \Lambda)$-graph. Form the $\frac{1}{2} K V$ sets of size 2 which consist of pairs of points which are joined in the graph. These sets are the blocks of a $P B I B D(2)$ with suitable parameters.

\section{Necessary conditions}

THEOREM 4. SUPpose there is a $(V, K, \Lambda)$-graph. Then there is an integer $m$ satisfying

$$
\begin{gathered}
m^{2}=K-\Lambda, \\
m \mid k, \\
V-1 \text { and } k^{-1} \text { have the same parity. }
\end{gathered}
$$

The above theorem has been proven by Bose and Shrikhande [4]. Their proof involves consideration of the matrix properties of the incidence matrix of the graph. We will show that the theorem follows immediately from the known results on $P B I B D(2) \mathrm{s}$. The theorem has also been derived by N. Sauer [8].

In [6] Connor and Clatworthy define two parameters $\Delta$ and $\eta$ associated with a $P B I B D(2)$ :

$$
\begin{aligned}
& \Delta=\left(p_{12}^{2}-p_{12}^{1}\right)^{2}+2\left(p_{12}^{2}+p_{12}^{2}\right)+1, \\
& n=\left[(v-1)\left(1+p_{12}^{1}-p_{12}^{2}\right)-2 n_{1}\right] / 2 \sqrt{\Delta} .
\end{aligned}
$$

They then prove the following Theorem:

LEMMA 5 [6, p. 110]. In a PBI B D (2) : 
(i) if $v$ is odd and $\Delta$ is not a perfect square, then $\Delta \equiv I(\bmod 4) ;$

(ii) if $v$ is odd and $\Delta$ is a perfect square, then $n$ is an integer;

(iii) if $v$ is even then $\Delta$ is a perfect square and $2 n$ is an odd integer.

Proof of Theorem 4. Suppose there is a $(V, K, N)$-graph. Then there is a $P B I B D$ (2) with parameters as in Theorem 3. For this design

$$
\begin{aligned}
& \Delta=4(K-\Lambda), \\
& \eta=-K / 2 \sqrt{ }(K-\Lambda) .
\end{aligned}
$$

Therefore the situation in $(i)$ of Lemma 5 cannot occur, so $\Delta$ is always a perfect square. Hence $(K-\Lambda)$ is always a perfect square; call it $\mathrm{m}^{2}$. This $m$ satisfies (4). In both ( $i i$ ) and ( $i i i)$ it is required that $2 n$ be an integer, so we have (5). The remaining conditions demanded by (ii) and (iii) are:

(ii) if $V$ is odd then $2 m \mid K$,

(iii) if $V$ is even then $\mathrm{Km}^{-1}$ is odd.

The first of these means that $\mathrm{Km}^{-1}$ is even. So, in either case, $\mathrm{Km}^{-1}$ has the same parity as $V-1$, and we have (6).

In [11] we derive another necessary condition for a $(V, K, \Lambda)$-graph: $V-2 K+\Lambda$ cannot equal 1 . This also follows from theorem 3 . From the parameters given in the Theorem, we derive (using (i))

$$
\begin{aligned}
& p_{22}^{1}=V-2 K+\Lambda, \\
& p_{22}^{2}=V-2 K+\Lambda-2 .
\end{aligned}
$$

By the definition of $p_{j k}^{i}$ it is clear that $p_{j k}^{i}$ cannot be negative if there is a pair of $i-t h$ associates in the design. So $p_{22}^{2}$ is negative only if all points are first associates - that is, the graph is complete. So $V-2 K+\Lambda \geqq 2$ except in the case of a complete graph, which satisfies $V-2 K+\Lambda=0$. So $V-2 K+\Lambda \neq 1$. 


\section{A two-parameter family of graphs}

Ray-Chaudhuri has constructed block designs by considering quadrics in finite projective spaces. He takes lines generating the quadric as blocks and points on the quadric as treatments. He derives the following theorem $[7$, p. 1181] by considereing a non-degenerate quadric in $P G(2 t, s)$, the 2t-dimensional space with co-ordinates in $G F(s)$.

THEOREM 6. If $t$ is a positive integer and $s$ is a prime power, there is a $P B I B D$ (2) with

$$
\begin{aligned}
& v=\left(s^{2 t}-1\right) /(s-1), \\
& n_{1}=s\left(s^{2 t-2}-1\right) /(s-1), \\
& p_{11}^{1}=(s-1)+s^{2}\left(s^{2 t-4}-1\right) /(s-1), \\
& p_{11}^{2}=\left(s^{2 t-2}-1\right) /(s-1) .
\end{aligned}
$$

Using (1) and (2) we find that this design satisfies

$$
\begin{aligned}
& n_{2}=s^{2 t-1}, \\
& p_{22}^{1}=s^{2 t-2}(s-1)=p_{22}^{2} .
\end{aligned}
$$

Hence we can use Theorem 2 to obtain

COROLLARY 7. If $s$ is any prime power and $t$ any positive integer, then there is a $(V, K, \Lambda)$-graph with parameters

$$
\left(\left(s^{2 t}-1\right) /(s-1), s^{2 t-1}, s^{2 t-2}(s-1)\right) \text {. }
$$

We shall refer to a graph with these parameters as being of type $D_{t}(s)$

REMARKS. 1. A graph of type $D_{t}(2)$ is also a graph of type $C_{2} t-1$ (in the notation of our paper [10]). Therefore we know of two constructions for graphs with parameters $\left(2^{2 t}-1,2^{2 t-1}, 2^{2 t-2}\right)$. It is not known whether the two graphs are isomorphic.

2. Several other families of partially balanced designs exist which yield graphs of type $D_{2}(s)$. This series is found by applying Theorem 2 to the designs in [5] or to either of the two classes of designs on p. 1182 of [7]. Again, it is not known whether the various graphs arising are isomorphic. 


\section{References}

[1] R.W. Ahrens and G. Szekeres, "On a combinatorial generalization of 27 lines associated with a cubic surface", J. Austral. Math. Soc. (to appear).

[2] R.C. Bose, "Strongly regular graphs, partial geometries and partially balanced designs", Pacific J. Math. 13 (1963), 389-419.

[3] R.C. Bose and W.H. Clatworthy, "Some classes of partially balanced designs", Ann. Math. Statist. 26 (1955), 212-232.

[4] R.C. Bose and S.S. Shrikhande, Grophs in which each pair of vertices is adjacent to the same number $d$ of other vertices (Institute of Statistics Mimeo Series No. 600.6, Consolidated University of North Carolina, 1969).

[5] Willard H. Clatworthy, "A geometrical configuration which is a partially balanced incomplete block design", Proc. Amer. Math. Soc. $5(1954), 47-55$.

[6] W.S. Connor and W.H. Clatworthy, "Some theorems for partially balanced designs", Ann. Math. Statist. 25 (1954), 100-112.

[7] D.K. Ray-Chaudhuri, "Application of the geometry of quadrics for constructing PBIB designs", Ann. Math. Statist. 33 (1962), 1175-1186.

[8] Norbert Saver, " $(v, k, \lambda)$-graphs" (Calgary International Conference on Combinatorial Structures and their Applications, Abstracts, University of Calgary, 1969).

[9] S.S. Shrikhande and N.K. Singh, "On a method of constructing symmetrical balanced incomplete block designs", Sankhyā, Ser. A 24 (1962), 25-32.

[10] W.D. Wallis, "Certain graphs arising from Hadamard matrices", Buzz. Austral. Math. Soc. 1 (1969), 325-331.

[11] W.D. Wallis, "A non-existence theorem for $(v, k, \lambda)$-graphs", J. Austraz. Math. Soc. (to appear).

La Trobe University,

Bundoora, victoria. 